\title{
28 Research Square \\ Anticoagulation Contributes to Antimicrobial Treatment of Lemierre Syndrome: A Case Report
}

Jie Ge

Changzhou First People's Hospital

Peipei Zhou

Changzhou First People's Hospital

\section{Yifei Yang}

Changzhou First People's Hospital

\section{Tianshu Xu}

Changzhou First People's Hospital

Xu Yang ( $\square$ nova_gejie@163.com )

Department of stomatology, Changzhou First People's Hospital, Juqian Street 185, Changzhou, Jiangsu, China

\section{Case report}

Keywords: Anticoagulation, Infectious Disease, Thromboembolism, Lemierre syndrome, case report

Posted Date: November 13th, 2020

DOl: https://doi.org/10.21203/rs.3.rs-104611/v1

License: (c) (i) This work is licensed under a Creative Commons Attribution 4.0 International License.

Read Full License 


\section{Abstract}

Background: Lemierre syndrome (LS) is characterized by multisystemic infection beginning in the oropharynx, thrombophlebitis of the internal jugular vein and thrombotic extension to the periphery. It is difficult to establish an evidence-based guideline for management of the disease and the use of anticoagulation therapy remains controversial.

Case presentation: A 61-year-old man suffered left neck swelling, odynophagia, and dyspnea. He underwent emergency surgery and received intravenous antimicrobial treatment. The primary infection was controlled on hospital day 5. However, he had a sudden increased WBC and decreased SpO on hospital day 6 . Further thoracic CT angiography revealed an intraluminal filling defect in the pulmonary artery on hospital day 8 . LS was diagnosed and then anticoagulation therapy was initiated. The WBC count maintained its peak value on hospital days 6-8 before anticoagulation therapy was initiated and decreased instantly after initiation.

Conclusions: Our case supported the opinion that anticoagulation therapy contributed to the antiinflammatory effect achieved by the LS treatment.

\section{Background}

Despite its nonnegligible annual morbidity of 1/100 000, Lemierre syndrome (LS) often goes undiagnosed due to nonspecific symptoms ${ }^{1,2}$. The most accurate definition of LS should include 1) opportunistic infection that originates in the oropharynx, mastoiditis, oral cavity and sinusitis, 2) isolation of Fusobacterium necrophorum and 3) thrombophlebitis of the internal jugular vein producing disseminated emboli to the periphery (often septic pulmonary emboli) ${ }^{2-4}$. However, as an obligate anaerobic bacterium, Fusobacterium necrophorum is notoriously difficult to culture. It requires a longer incubation period than other bacteria, and cultures can be false negative if antibiotics are administered before sample collection. Although Fusobacterium necrophorum may be undetected or other pathogenic bacteria may be detected, a clinical diagnosis of LS is still valid ${ }^{5}$. Delayed treatment of LS can lead to multisystemic organ dysfunction, potential end-stage organ damage or death. Systemic emboli and septic pulmonary emboli can also be life threatening. Therefore, early diagnosis and treatment of LS are vital. The key tools of physicians are antibiotic therapy, anticoagulation therapy and internal jugular vein surgery ${ }^{2}$. The use of anticoagulation therapy remains controversial, and it is unclear whether anticoagulation treatment will promote the resolution of thrombosis. Here, we report a case that demonstrates that anticoagulation therapy is supportive of antimicrobial treatment in LS.

\section{Case Presentation}

A 61-year-old man presented to the oral and maxillofacial surgery service with a 5-day history of left neck swelling, odynophagia, and dyspnea without chest pain or cough. He reported a history of 7-day odontalgia and 3-day intravenous cefotiam ( $1 \mathrm{~g}$ every $12 \mathrm{~h}$ ) and metronidazole (500 mg every $8 \mathrm{~h}$ ) 
treatment. He was healthy at baseline. He was married and had one daughter. He had no known allergies. At the time of admission, the patient had an oxygen saturation (SpO) of $98 \%$ while breathing ambient air. Laboratory data revealed a white blood cell count (WBC) of $20.45 \times 10^{9} / \mathrm{L}$, procalcitonin (PCT) of 1.142 $\mathrm{ng} / \mathrm{mL}$ (Fig. $1 \mathrm{~A}$ ), random blood glucose of $16.02 \mathrm{mmol} / \mathrm{L}$ and glycosylated hemoglobin of $8.7 \%$. Computed tomography (CT) of the neck revealed diffusive swelling combined with pneumatosis (Fig. 1 $B)$. The patient was then diagnosed with cervical necrotizing fasciitis.

He underwent emergency incision and drainage surgery and received empirical treatment with intravenous imipenem-cilastatin sodium ( $1 \mathrm{~g}$ every $8 \mathrm{~h}$ ) and metronidazole (500 mg every $8 \mathrm{~h}$ ). His blood glucose was controlled by insulin. Anaerobic cultures of pyogenic fluids and blood were obtained on admission and were sterile 5 days later, which may have been due to the antimicrobial treatment before admission. After five days of antimicrobial treatment (hospital day 5), the patient's WBC gradually decreased to $18.12 \times 10^{9} / \mathrm{L}$ (Fig. 1 A). However, he had an SpO of $80 \%$ with ambient air and $96 \%$ with oxygen inhalation ( $5 \mathrm{~L} / \mathrm{min}$ ) on the sixth hospital day. Laboratory data showed that his WBC count increased to $21.77 \times 10^{9} / \mathrm{L}$, while his PCT level decreased to $0.211 \mathrm{ng} / \mathrm{mL}$ (Fig. 1 A). A repeat CT scan revealed that the neck infection was improved (Fig. 1 B) and that the pulmonary infection did not progress (Fig. $1 \mathrm{C}$ ). Further thoracic CT angiography revealed an intraluminal filling defect in the pulmonary artery on the eighth hospital day (Fig. $1 \mathrm{D}$ ). This manifestation was considered septic pulmonary emboli derived from the internal jugular vein, which was very close to the infection focus (Fig. $1 \mathrm{E})$. LS was diagnosed.

Anticoagulation therapy was performed with low molecular weight heparin (4250 U every $12 \mathrm{~h}$ ) for the following four days. His WBC gradually decreased to $17.87 \times 10^{9} / \mathrm{L}$ (Fig. 1 A). Low molecular weight heparin was replaced with warfarin ( $3 \mathrm{mg}$ every day) on hospital day 12 . The international normalized ratio (INR) was monitored constantly and controlled between 2-2.5. The paitent's WBC count decreased to $8.67 \times 10^{9} / \mathrm{L}$ on hospital day 17 and remained normal on the following days (Fig. 1 A). Chest CT reexamination showed that the pulmonary infection was cured on hospital day 22 (Fig. 1 C). He was discharged from the hospital on hospital day 24 and continued anticoagulation therapy. When he returned for a follow-up visit at the twentieth week, the embolus was dissolved (Fig. 1 D), and anticoagulation therapy was discontinued. No hemorrhagic manifestation was found during anticoagulation therapy.

\section{Discussion And Conclusions}

In this case, the primary infection was controlled on hospital day 5 , as proven by CT evaluation and decreased WBC and PCT levels. The sudden increase in WBC count and decrease in SpO on hospital day 6 were considered to be caused by newly formed septic pulmonary emboli. However, three days were required for diagnosis of LS due to a lack of experience. As a result, anticoagulation therapy was not initiated until hospital day 8 , although clinical manifestations appeared on day 6 . Interestingly, the WBC count maintained its peak value on hospital days 6-8 before anticoagulation therapy was initiated and 
decreased instantly after initiation. This kind of change demonstrated that anticoagulation therapy contributed to the antiinflammatory effect achieved by the LS treatment.

In a review of 137 cases of LS, the authors found that 4-6 weeks of carbapenem or piperacillin/tazobactam combined with metronidazole were optimal in terms of antiinflammatory therapy, but the reason for the choice of such a duration was not explained in any of the included papers ${ }^{5}$. Our antiinflammatory treatment was performed until the WBC counts remained normal for one week and clinical symptoms improved. The duration was 24 days in total and 16 days after anticoagulation therapy proceeded, which is much shorter than the average. However, it is still unknown whether anticoagulation therapy is essential.

In the past few decades, evidence remains poor regarding the management of LS, particularly regarding its potentially life-threatening thromboembolic complications. Consequently, physicians must make their decisions based on small case series or anecdotal cases when facing uncommon thromboembolic conditions, such as LS. Physicians who are opposed to anticoagulant treatment confirm that the thrombus is caused by an infection process and will be resolved at the same time when the infection is resolved. In contrast, some authors confirm that anticoagulant therapy may reduce the morbidity and mortality of serious complications, such as cavernous sinus thrombosis or pulmonary embolism. Theoretically, a septic thrombus sequesters bacteria and creates a barrier to antibiotic penetration. When the thrombus is dissolved by anticoagulants, the bacteria are exposed to a higher concentration of antibiotics, increasing accessibility ${ }^{6}$. Our case supports this hypothesis precisely. Unfortunately, randomized controlled trials are impractical to investigate the use of anticoagulation therapy in the treatment of LS due to the rarity of this illness.

A recent European collaborative study performed an individual patient-level analysis of 712 cases published globally from 2000 to $2017^{1}$. The authors could not find disease-specific elements regarding anticoagulation therapy safety and drew the conclusion that therapeutic anticoagulation is indicated for LS if there are no contraindications. Nevertheless, the authors did not provide definitive guidelines for the duration of anticoagulation. According to the American College of Chest Physicians guidelines for provoked thrombotic events, anticoagulation therapy is recommended for a duration of at least 3 months 7 . Our patient received a treatment duration of 20 weeks because his follow-up visit was delayed by the outbreak of COVID-19.

In conclusion, therapeutic anticoagulation may be considered in the management of LS, while further research is needed to evaluate the different outcomes between patients treated with anticoagulants and antibiotics and patients with antibiotics alone.

\section{Patient perspective}

I'm the daughter of the patient. We are grateful to the doctors' help in Changzhou First People's Hospital and happy to share the treatment experience of my farther. 


\section{List Of Abbreviations}

LS Lemierre syndrome

SpO oxygen saturation

WBC white blood cell count

PCT procalcitonin

CT computed tomography

INR international normalized ratio

\section{Declarations}

\section{Ethics approval and consent to participate}

Institutional review board approval was not required for this case report. Informed consent was obtained from the patient.

\section{Consent for publication}

Consent for publication was obtained from the patient.

\section{Availability of data and materials}

The datasets generated and/or analysed during the current study are not publicly available due to patient privacy protection but are available from the corresponding author on reasonable request.

\section{Competing interests}

The authors declare that they have no competing interests.

\section{Funding}

This work was supported by Changzhou Science and Technology Bureau (CN) (grant number CJ20180060).

\section{Authors' contributions}

JG and XY performed the literature review and drafted the manuscript. PPZ and YFY obtained the image data. TSX reviewed the report and provided important revisions. All authors read and approved the final manuscript.

\section{Acknowledgements}


Not applicable.

\section{References}

1. Valerio L, Zane F, Sacco C, Granziera S, Nicoletti T, Russo M, et al. Patients with Lemierre syndrome have a high risk of new thromboembolic complications, clinical sequelae and death: an analysis of 712 cases. Journal of internal medicine. 2020 May 22. DOI: 10.1111/joim.13114.

2. Osowicki J, Kapur S, Phuong LK, Dobson S. The long shadow of lemierre's syndrome. The Journal of infection. 2017 Jun;74 Suppl 1:S47-S53. DOI: 10.1016/S0163-4453(17)30191-3

3. Schulman S. Lemierre syndrome - treat with antibiotics, anticoagulants or both? Journal of internal medicine. 2020 May 18. DOI: 10.1111/joim.13100

4. Walkty A, Embil J. Lemierre's Syndrome. The New England journal of medicine. 2019 Mar 21;380(12):e16. DOI: 10.1056/NEJMicm1808378

5. Johannesen KM, Bodtger U. Lemierre's syndrome: current perspectives on diagnosis and management. Infection and drug resistance. 2016;9:221-7. DOI: 10.2147/IDR.S95050

6. Campo F, Fusconi M, Ciotti M, Diso D, Greco A, Cattaneo CG, et al. Antibiotic and Anticoagulation Therapy in Lemierre's Syndrome: Case Report and Review. J Chemother. 2019 Feb;31(1):42-8. DOI: 10.1080/1120009X.2018.1554992

7. Kearon C, Akl EA, Ornelas J, Blaivas A, Jimenez D, Bounameaux H, et al. Antithrombotic Therapy for VTE Disease: CHEST Guideline and Expert Panel Report. Chest. 2016 Feb;149(2):315-52. DOI: 10.1016/j.chest.2015.11.026

\section{Figures}




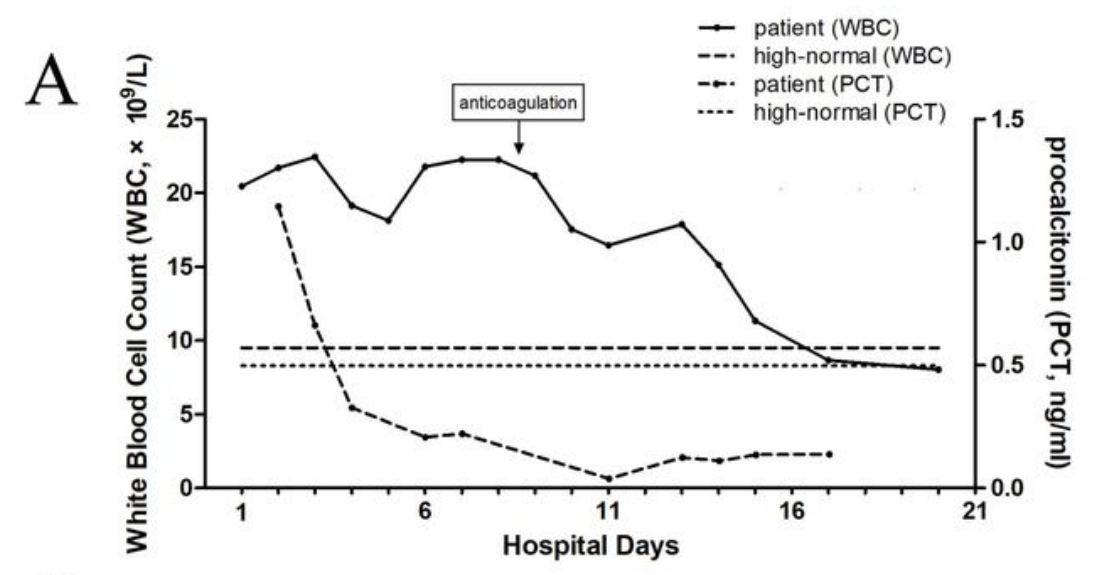

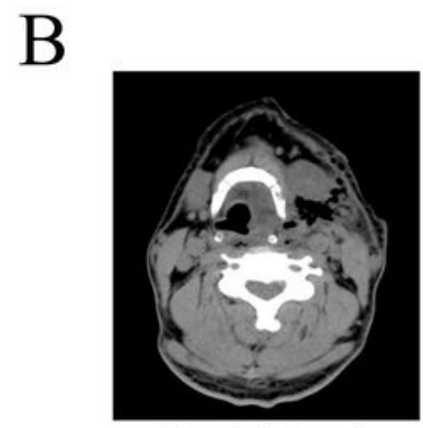

Hospital Day 1

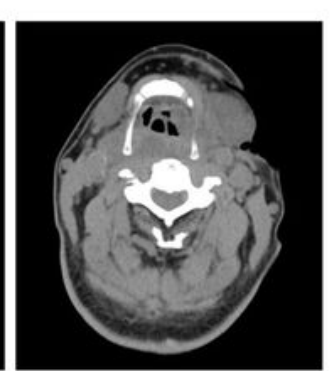

Hospital Day 7

C

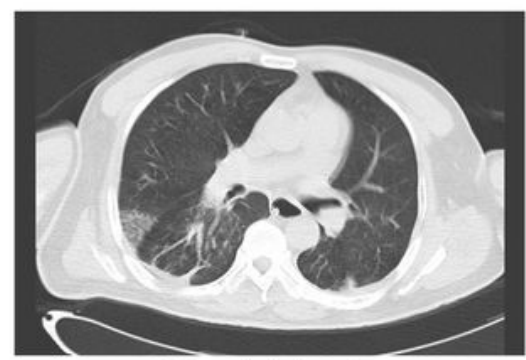

Hospital day 3

D

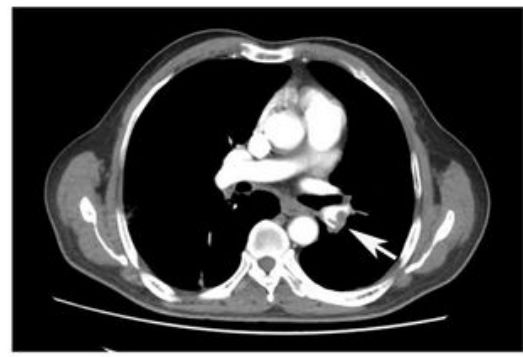

Hospital Day 8

E

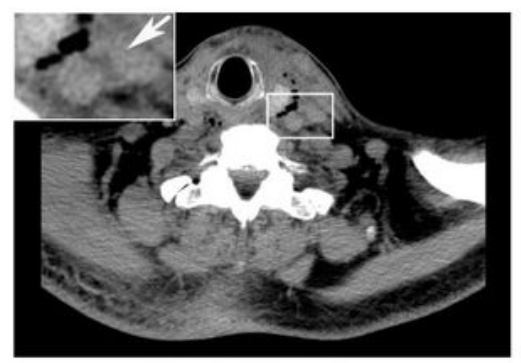

Hospital Day 1

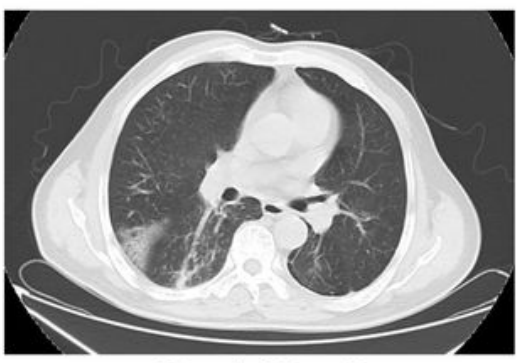

Hospital Day 7

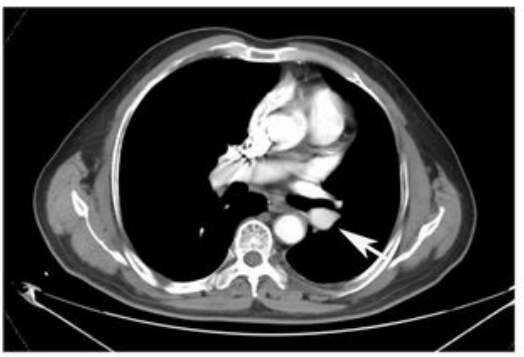

Follow-up Visit

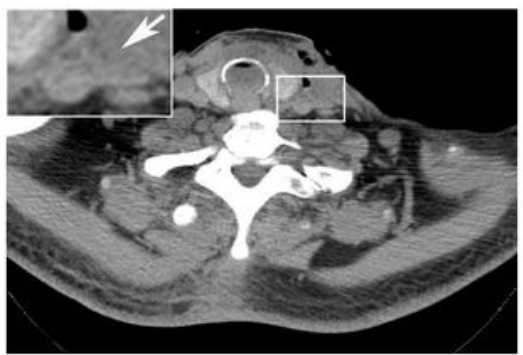

Hospital Day 7

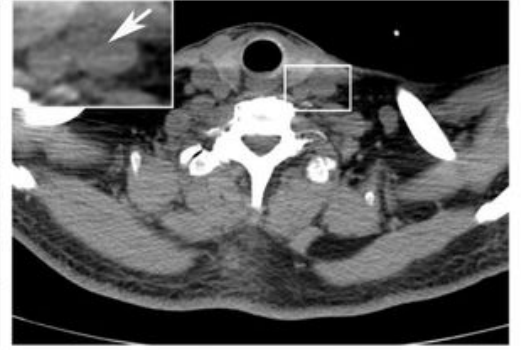

Follow-up Visit

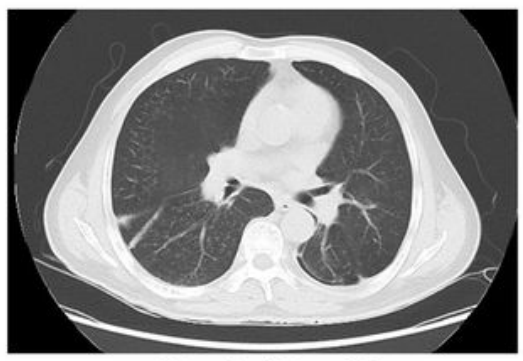

Hospital Day 22

\section{Figure 1}

The patient's clinical data. A: WBC count and PCT level changes during the hospital stay. B: CT manifestation changes in the neck. C: CT manifestation changes in chest. D: CT angiography changes in the pulmonary artery. White arrow: pulmonary emboli in the "Hospital Day 8" image; pulmonary emboli disappeared in the "Follow-up Visit" image. E: CT manifestation changes in the internal jugular vein 
infection. White arrow: infections next to the internal jugular vein in "Hospital Day 1" and "Hospital Day 7" images; the infection disappeared in the "Follow-up Visit" image.

\section{Supplementary Files}

This is a list of supplementary files associated with this preprint. Click to download.

- CAREChecklist.jpg

- CAREChecklist.jpg 